\title{
La ingeniería documental: su posible aproximación académica
}

\author{
Por Ricardo Eíto-Brun
}

\begin{abstract}
Resumen: El diseño de tipos de documentos es una actividad generalizada en la práctica profesional desde hace años. Sin embargo, la generalización del lenguaje XML como medio para codificar e intercambiar documentos a través de redes y la aparición de distintos métodos para codificar las características de los tipos de documentos (esquemas XML, RelaxNG, etc.) y sus metadatos descriptivos y administrativos ha hecho manifiesta la necesidad de contar con una aproximación más estructurada para formalizar el diseño de tipos de documentos y su implementación mediante estándares XML. Esto se une a los requisitos de la nueva aproximación tecnológica para la integración de aplicaciones software - los llamados servicios web o arquitecturas SOA (Service Oriented Architecture) - que basan la interacción entre sistemas en el intercambio de documentos XML cuya estructura debe definirse con precisión. La principal aproximación teórica a este problema lo encontramos en el trabajo académico de Robert J. Glushko, con el nombre de Ingeniería Documental. Esta aproximación, que se describe brevemente en el texto, incluye las actividades necesarias para la definición de tipos de documentos y su utilización como interfaces entre sistemas informáticos heterogéneos en arquitecturas SOA. Constituye una aproximación formal y académica a una de las principales oportunidades para la práctica profesional actual.
\end{abstract}

Palabras clave: XML, Tipos de documentos, Esquemas XML, Ingeniería documental, Servicios web, SOA (Arquitectura orientada a servicios).

\section{Title: Document engineering: potential academic applications}

Abstract: Document design has long been a standard task for information professionals. Nonetheless, the use of XML to encode and transfer electronic documents across networks and the development of various methods to describe the characteristics of various document types (XML schemas, RelaxNG, etc.) and their metadata have made clear the need for a more formal, structured approach to the design of various document types and their implementation using XML standards. This insight, combined with the requirements of new technological approaches to integrating software applications - web services or service-oriented architecture (SOA) - that rely on XML for document exchange across platforms, results in a need for documents whose structure is precisely defined. The work of Robert J. Glushko, specifically document engineering, provides the primary theoretical approach to this problem. Document engineering includes the activities necessary for the design of document types and their use as interfaces between systems in SOA environments. His work offers a formal, academic approach to what is currently one of the most interesting opportunities for information professionals.

Keywords: XML, Document Type, XML Schemas, Document Engineering, Web Services, SOA (Service Oriented Architecture).

Eíto-Brun, Ricardo. "La ingeniería documental: su posible aproximación académica”. En: El profesional de la información, 2007, julio-agosto, v. 16, n. 4, pp. 490-496.

DOI: $10.3145 /$ epi.2007.sep.12

A MEDIDA QUE SE HA GENERALIZADO LA ADOPCIÓN de tecnologías informáticas y el uso de redes de comunicaciones en las organizaciones, se ha hecho manifiesta la necesidad de adoptar formatos comunes para el intercambio de datos y establecer pautas que favorezcan el uso de formatos de datos normalizados. Esta práctica es la mejor garantía para asegurar la integridad en el intercambio de información y hacer que los costes de estas operaciones no se conviertan en un obstáculo para su adopción.

Inicialmente la normalización documental estuvo centrada en el intercambio de documentos comerciales, con las distintas generaciones del EDI (Electronic Data Interchange) y formatos clásicos como X12 o Edifact. En los últimos años, el alcance de estas actividades de normalización se ha ampliado a otras áreas como la gestión sanitaria, la educación, los recursos humanos o la actividad industrial. Ejemplos de esto son iniciativas como los lenguajes $H L 7, x b r l$ o $H R-X M L$. En todos estos escenarios, la documentación electrónica constituye el medio que hace posible la colaboración entre organizaciones basada en un intercambio de información digital. 
Pero la normalización no sólo implica la necesidad de establecer acuerdos sobre el formato y el contenido de los documentos, sino también sobre su utilización prevista y los procesos de trabajo de los que forman parte. De hecho, son numerosas las metodologías de representación y optimización de procesos administrativos que basan la descripción de éstos en los documentos que se transmiten entre unidades organizativas. Así, el esfuerzo normalizador abarca distintos aspectos:

- Los técnicos relacionados con la transmisión física de los mensajes a través de redes de comunicaciones.

- Los puramente documentales vinculados a los datos que se deben incluir en los documentos y a la forma de organizarlos

- los organizativos, en relación a los procesos en los que se usarán los documentos, el orden en el que deben intercambiarse y las dependencias que existen entre ellos.

La obra de Robert Glushko es en la actualidad la aproximación académica que mejor refleja los problemas de la normalización documental en la colaboración electrónica entre organizaciones. Glushko es profesor de la School of Information Management \& Systems de la Universidad de Berkeley, California y fundador de la empresa Veo System, adquirida en 1999 por CommerceOne, uno de las principales compañías dedicadas al

\footnotetext{
"Ingeniería documental

se refiere a las distintas técnicas que se utilizan para establecer modelos que hagan posible el intercambio de documentos entre organizaciones"
}

diseño de software para el comercio electrónico.

Este autor propuso la utilización del término ingeniería documental para referirse a las distintas técnicas y tecnologías que se utilizan para establecer modelos que hagan posible el intercambio de documentos entre organizaciones. $\mathrm{Su}$ obra sobre este tema está formada por diversos artículos y contribuciones a congresos, así como de manera especial por el libro escrito en colaboración con Tim McGrath (Glushko-Mcgrath, 2005) , en el que se analiza y describe el alcance de esta disciplina desde una perspectiva conceptual y práctica.

Concretamente Glushko la define como "la disciplina dedicada a especificar, diseñar e implementar los documentos electrónicos que se usarán en los intercambios de información en internet para solicitar la ejecución de procesos de negocio, o para devolver los resultados obtenidos por la ejecución de estos procesos a través de servicios web". Otra definición propuesta por el autor señala que "la ingeniería documental estudiaría los métodos de análisis y diseño, y los modelos formales para describir la información requerida por los procesos de negocio, así como la secuencia o coreografía mediante la cual estos procesos se coordinan" (Glushko, 2003).

Vemos que el término agrupa distintas técnicas: diseño de tipos de documentos, análisis de procesos y modelos de transacciones, así como los procedimientos necesarios para el intercambio de información y documentos a través de la Red. Podríamos decir que son tres sus objetivos:

- Modelar procesos y transacciones entre empresas, y representarlos en un formato que haga posible su procesamiento automatizado.

- Modelar la estructura de los documentos que se intercambian en esos procesos. Normalmente mediante $\mathrm{xml}$.

- Implementar soluciones informáticas capaces de ejecutar y auditar estas transacciones en entorno internet, garantizando la autenticidad, confidencialidad y seguridad de los datos.

Todos ellos se corresponden con tres prácticas habituales en muchas organizaciones: diseño de procesos, diseño de tipos documentales mediante esquemas xml e implementación de servicios web. Un punto que debemos aclarar es que la ingeniería documental no debe interpretarse como la disciplina que ha dado origen a estas tres técnicas (esquemas xml, modelado de procesos y servicios web). Lo interesante de la aproximación de Glushko es el hecho de reunir distintas técnicas que han surgido y evolucionado de forma independiente en un marco teórico común, llamado a facilitar la interpretación y el diseño de soluciones para entornos de colaboración complejos.

\section{Los principios de la ingeniería documental}

Está vinculada al comercio electrónico y a la colaboración entre empresas a través de internet. Actualmente, la Red es un canal consolidado en la venta a particulares (el llamado B2C o business to consumer) y en la promoción de productos y servicios. Sin embargo, su utilización como un canal para el comercio entre empresas sigue siendo una asignatura pendiente para muchas organizaciones, sin que se hayan materializado todas las expectativas y su potencial real. En este sentido, resulta ilustrativa la escasa aceptación de numerosas iniciativas $B 2 B$, que ha demostrado una falta de madurez por parte del mercado para aceptar y comprender unos modelos de negocio que antes o después están llamados a generalizarse. 


\section{Modelado de datos}

Un modelo de datos es una colección de conceptos bien definidos matemáticamente que ayudan a expresar las propiedades estáticas y dinámicas de una aplicación.

Propiedades estáticas: entidades (u objetos), propiedades (o atributos) de esas entidades, y relaciones entre esas entidades.

Propiedades dinámicas: operaciones sobre entidades, sobre propiedades o relaciones entre operaciones.
Reglas de integridad sobre las entidades y las operaciones (por ejemplo, transacciones).

Así, un modelo de datos se distingue de otro por el tratamiento que da a estas tres categorías. El resultado de un modelado de datos es una representación que tiene dos componentes: las propiedades estáticas se definen en un esquema y las propiedades dinámicas se definen como especificaciones de transacciones, consultas e informes.

\section{"La crisis de las 'punto com' y el repliegue de la inversión supuso un freno a la generalización de los entornos de colaboración electrónica"}

La crisis de las "punto com" y el repliegue de la inversión supuso un freno a la generalización de los entornos de colaboración electrónica. Sin embargo, el ahorro que supone el intercambio de documentos comerciales a través de internet, la posibilidad de optimizar procesos e interactuar con nuevos socios, unidos al nivel de seguridad que ofrecen las actuales tecnologías, sitúan a la ejecución de transacciones a través de internet entre los objetivos prioritarios de la mayor parte de las organizaciones.

La siguiente pregunta que tendríamos que plantearnos es, ¿cómo contribuye la visión académica de Glushko? Las pautas y principios que caracterizan la propuesta de este autor nos pueden ayudar a dar una respuesta; éstos incluyen:

- Intercambio de información y coordinación de procesos.

- Normalización de procesos.

- Carácter modular y reutilización de documentos.
- Utilización del lenguaje xml.

- Análisis y diseño de documentos frente al modelado de datos.

- Documentos orientados a transacciones.

- Clasificación de productos y servicios.

\section{Intercambio de información y coordinación de procesos}

Los escenarios $B 2 B$ se sitúan próximos a los entornos de colaboración que caracterizan el concepto de "empresa virtual". En este modelo, distintas compañías colaboran en el diseño, fabricación o comercialización de un producto; cada una de ellas ofrece al resto de socios un elevado nivel de especialización en alguna de las tareas que se deben ejecutar para completar el ciclo de vida del producto o servicio y facilitar su comercialización.

El modelo de empresa virtual se basa en un intercambio continuo de información entre socios. La noción de "cadena de suministro" tradicionalmente orientada hacia el intercambio de bienes físicos, se complementa con una orientación centrada en el intercambio de información. Si el proceso se hace de forma óptima permitirá obtener los beneficios derivados de una gestión eficiente de inventarios, previsión de necesidades futuras, etc.

El concepto de colaboración entre empresas aplicando las tecnologías de la información y el de "empresa virtual" es anterior a internet. Sin embargo, también es cierto que la Red hizo posible que cualquier empresa, sin necesidad de afrontar costosas inversiones, pudiese plantearse su participación en un sistema de colaboración de este tipo, o llegar a articularlo.

El ejemplo pionero en la colaboración entre empresas y automatización de la cadena de suministro es la iniciativa que puso en marcha Procter \& Gamble en 1987 con la empresa Wal-Mart para la gestión de inventarios y aprovisionamiento ${ }^{1}$. Se basó en la automatización de la cadena de suministro $(C P R F$ $o$ Collaborative Planning, Forecasting and Replenishment en inglés), de forma que ambas empresas compartían un proceso común facilitado por una tecnología desarrollada a partir de un sistema de $I B M^{2}$.

La base era la capacidad de compartir información entre proveedor y suministrador y automatizar el intercambio de documentos (estados de inventario, órdenes de compra, facturas, etc.). Los resultados se tradujeron en un mejor nivel de servicio y en la reducción de costes derivados de excesos y defectos 


\section{Proceso de negocio}

Es un conjunto de tareas relacionadas lógicamente llevadas a cabo para lograr un resultado de negocio definido. Cada proceso de negocio tiene sus entradas, funciones y salidas. Las entradas son requisitos que deben tenerse antes de que una función pueda ser aplicada. Cuando una función es aplicada a las entradas de un método, tendremos ciertas salidas resultantes.

Un proceso de negocio es una colección de actividades estructurales relacionadas que producen un valor para la organización, sus inversores o sus clientes. Es, por ejemplo, el proceso a través del que una organización realiza sus servicios a sus clientes.

Un proceso de negocio puede ser parte de un proceso mayor que lo abarque o bien puede incluir otros procesos de negocio que deban ser incluidos en su función. En este contexto un proceso de negocio puede ser visto a varios niveles de granularidad. El enlace entre procesos de negocio y generación de valor lleva a algunos practicantes a ver los procesos de negocio como los flujos de trabajo con que se efectúan las tareas de una organización.

Los procesos de negocio pueden ser vistos como un recetario para hacer funcionar un negocio y alcanzar las metas definidas en la estrategia de negocio de la empresa.

\section{Tipos principales}

1. Centrales: dan el valor al cliente, son la parte principal del negocio. Por ejemplo, "Repartir mercancías"
2. De soporte: Por ejemplo, "Contabilidad", "Servicio técnico".

Los procesos de negocio consisten en subprocesos, decisiones y actividades.

Un subproceso es parte un proceso de mayor nivel que tiene su propia meta, propietario, entradas y salidas.

Las actividades son partes de los procesos de negocio que no incluyen ninguna toma de decisión ni vale la pena descomponer (aunque ello sea posible). Por ejemplo, "Responde al teléfono", "Haz una factura"

Un proceso de negocio es usualmente el resultado de una Reingeniería de Procesos. El modelado de procesos es usado para capturar, documentar y rediseñar procesos de negocio.

En la época de Taylor un operario realizaba una tarea específica, pero luego se cambió esa perspectiva hacia los trabajos realizados por un equipo, teniendo en cuenta el cliente que es quien fija los resultados.

Esto facilita el acercamiento y el acuerdo con los clientes, aumenta la motivación de los empleados y mejora la respuesta a los cambios del contexto.

Para aplicar los procesos se deben tener claras las tareas, la estructura jerárquica y existir una tendencia a la interacción y la comunicación.

Fuente: Wikipedia

http://es.wikipedia.org/wiki/Proceso_de_ negocio en inventario, así como costes administrativos. Aparte, la literatura recoge numerosos casos similares, en los que se establece una cadena de suministro automatizada que une los puntos de venta con los sistemas de información de los proveedores; a día de hoy se trata de una práctica habitual y generalizada en distintos sectores industriales.

En este contexto, las empresas que colaboran en uno de estos en- tornos deben establecer y aceptar unas pautas comunes que garanticen el intercambio de información y su procesamiento automatizado. Estos principios se han centrado en la implementación de una infraestructura técnica para el intercambio de datos y en la definición de tipos de documentos con una estructura, formato y contenido pactado de antemano por los participantes. En la visión de Glushko, los documentos se convierten en las interfaces entre los procesos internos que ejecutan las empresas que participan en un entorno de colaboración.

Tanto las tecnologías de la información actuales como internet nos permiten definir modelos de colaboración más avanzados que el mero intercambio de documentos entre un proveedor y un cliente, como por ejemplo los mercados virtuales en internet que podrían servir para crear y gestionar empresas vir- 
tuales resultado de la colaboración (por un espacio de tiempo limitado) de distintos socios que se identifican y reúnen para dar respuesta a un proyecto específico planteado por un cliente. Estos modelos de subcontratación electrónica se han descrito en la literatura (Nayak, 2001), como la "capacidad de formar, gestionar y desarticular una empresa virtual en respuesta a una oportunidad determinada"; de esta forma, las empresas que actúan como proveedores, pueden superar sus límites o restricciones (falta de inventario, de competencias, etc.) mediante la colaboración temporal con otro socio que pueda cubrir sus carencias.

Estos modelos de organización (también descritos por Thomas Malone en su libro The future of work) son similares a la forma en la que se organizan los equipos de trabajo encargados de hacer una película, o en la forma en que se articulan proyectos informáticos o de consultoría, en los que una empresa cliente subcontrata equipos de distintas empresas especializados en un área específica, que colaborarán de manera conjunta para diseñar la solución o producto final.

En la propuesta de Nayak, las dificultades que afrontan las compañías para articular estas "empresas o equipos virtuales" son motivo de importantes pérdidas de oportunidades de negocio. La capacidad de gestionar un entorno virtual donde los clientes pudiesen publicar sus necesidades, y las empresas suministradoras fuesen capaces de identificar socios potenciales de forma automatizada eliminaría la dificultad para identificar socios y permitiría articular equipos de trabajo virtuales de forma rápida y efectiva. Nuevamente, la automatización de este proceso pasa, forzosamente, por la capacidad de describir de una forma estándar las capacidades que exige el proyecto propuesto por el cliente, las de los distintos provee- dores, y la capacidad de tramitar estos acuerdos en línea (dejando a parte otros aspectos no menos importantes, como los mecanismos capaces de garantizar la confianza entre socios potenciales antes de que se cierre un acuerdo o propuesta de trabajo común).

\section{Normalización de procesos}

La ingeniería documental parte de procesos de negocio para identificar transacciones y tipos documentales. Los procesos de negocio actúan bien como consumidores, o como productores de documentos que a su vez pueden ser utilizados por otros procesos de negocio.

Uno de sus objetivos como disciplina práctica es lograr representar y describir los patrones o modelos genéricos que guían el intercambio de documentos en un proceso determinado. Para que el traspaso de información entre empresas sea posible, los socios que participan en un proceso de colaboración deben haber alcanzado un acuerdo previo en la forma en la que se va a completar.

Sin embargo, para alcanzar un modelo que permita una mayor flexibilidad en la ejecución de procesos de negocio entre empresas, la ingeniería documental no sólo considera la posibilidad transmitir documentos; una opción más avanzada y que abre posibilidades adicionales es la de traspasar los datos junto con las especificaciones que detallarán el proceso que debe seguirse para tramitar dicha información. Considerando que el objetivo de cualquier transacción de comercio electrónico es que la información sea gestionada de forma desatendida por una aplicación informática, este modelo añade un nuevo requerimiento para estas aplicaciones, ya que tendrán que ser capaces de gestionar un mayor nivel de abstracción y de responder a una petición procedente de inter-

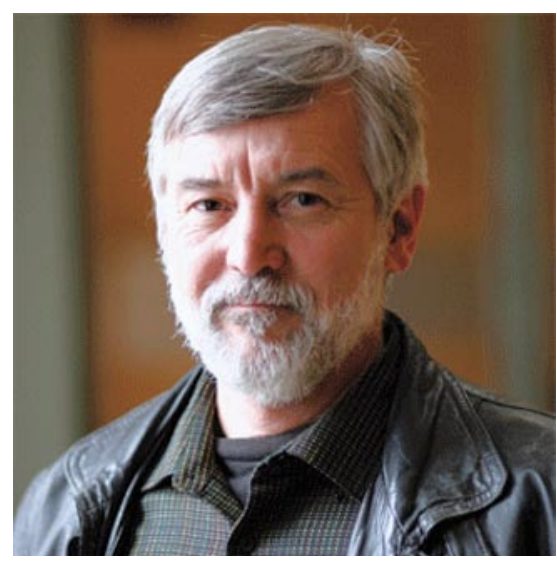

Robert J. Glushko, http://people.ischool. berkeley.edu/ glushko/

net de una u otra forma dependiendo del proceso especificado por la organización solicitante.

Obviamente, para que esto sea posible es imprescindible un conocimiento previo por parte de la empresa que inicia la transacción de los servicios que ofrece aquella a la que dirige la solicitud. Los sistemas de directorio basados en estándares como UDDI o ebXML permiten obtener la descripción de los servicios que ofrece cada organización así como la forma de invocarlos.

La propuesta inicial de Glushko abre importantes posibilidades para el estudio de la definición de procesos de negocio en forma de orquestaciones de servicios $w^{3} b^{3}$.

\section{Carácter modular y reutilización de documentos}

La reutilización de documentos $\mathrm{y}$ datos en distintos procesos y transacciones es otro de los objetivos que se tratan de satisfacer. La creación de repositorios de componentes comunes (que se podrán ensamblar en distintos tipos de documentos) es el principal mecanismo utilizado para permitir la reutilización y asegurar una mayor comprensión de los distintos tipos de documentos (un sistema informático que conozca la semántica y estructura definida para los componentes comunes 
será capaz de interpretar al menos parcialmente un documento que no haya procesado anteriormente $)^{4}$.

La base del diseño de documentos es su carácter modular y el uso de "componentes comunes". Esta pauta, que encontramos en $e b X M L$, $U B L$, incluso en el EDI tradicional, se basa en identificar y abstraer "fragmentos" o "estructuras de datos" que suelen aparecer en distintos tipos de documentos con una semántica similar. Por ejemplo, una “dirección de envío" es un fragmento o una estructura que se repite y utiliza en múltiples documentos: facturas, albaranes, pedidos, etc. Mantener un repositorio de componentes comunes y definir la forma en la que éstos deben ensamblarse para formar documentos completos agiliza la creación de nuevos tipos de documentos y permite responder con flexibilidad a demandas desconocidas de antemano.

\section{El protagonismo del lenguaje xml}

$\mathrm{Xml}$ ha hecho posible el intercambio de información estructurada a través de internet. Este lenguaje (junto con otras especificaciones relacionadas como los esquemas $\mathrm{xml}$, las transformaciones XSLT y los servicios web) constituye la base técnica de la ingeniería documental. Además permite la representación explícita del significado que tiene cada fragmento en un documento. La utilización del marcado descriptivo o generalizado (con el fin de intercalar marcas que permiten diferenciar $\mathrm{y}$ hacer explícita la estructura y el contenido informativo de un documento) hace

\section{"Xml ha hecho posible el intercambio de información estructurada a través de internet"}

que los documentos sean fácilmente procesables por cualquier aplicación informática.

Aunque el modelado de procesos y el diseño de tipos documentales se viene aplicando con anterioridad a la definición de xml, solamente la facilidad que caracteriza el procesamiento de datos $\mathrm{xml}$ y la adopción masiva por parte de la industria hacen posible un planteamiento como el que estamos describiendo, en el que no sólo los documentos, sino también las especificaciones sobre procesos de negocio y las capacidades técnicas de las organizaciones se codifican, transfieren e interpretan como datos $\mathrm{xml}$.

\section{Análisis y diseño de documentos frente al modelado de datos}

En lo referente al diseño de tipos de documentos (y su implementación mediante esquemas $\mathrm{xml}$ ), la ingeniería documental debe combinar dos tradiciones: el análisis y diseño de documentos propio de los lenguajes de marcas derivados de sgml, y las técnicas de modelado de datos para el diseño de bases de datos relacionales.

Glushko señala cómo el análisis de documentos se basa en alcanzar un modelo de documento a partir de una colección de documentos existentes, que comparten una estructura y unas características comunes. Esta abstracción establecerá unas reglas relativas a la estructura que deben compartir todos los que pertenezcan a un mismo tipo: qué secciones tendrán, el orden de éstas, cómo podrán anidarse unas dentro de otras, etc. Ésta ha sido una práctica habitual en el área de la edición electrónica, gestión de publicaciones y contenidos.

Por otra parte, el modelado de datos busca una representación de las distintas entidades que forman parte de un dominio o espacio que

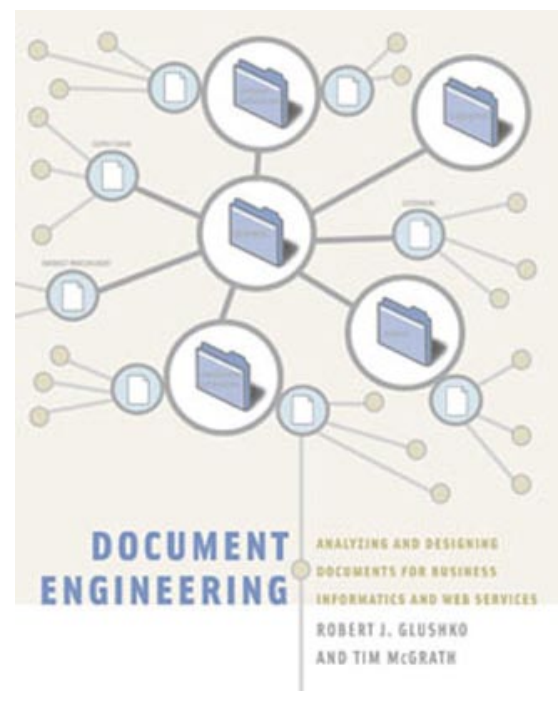

se pretende representar en una base de datos relacional. El modelo resultante describirá esas entidades mediante información estructurada, y las relaciones que existen entre ellas en el mundo real.

La distinción tradicional entre información estructurada (propia del modelado de datos y de las bases de datos relacionales) e información no estructurada (documentos), se hace más tenue y en parte se diluye con el lenguaje xml. La evolución de xml (y concretamente la posibilidad de usar esquemas xml para diseñar tipos de documentos en lugar de dtd) permite representar estructuras de datos con el nivel de precisión y detalle característico del modelado de datos relacional.

Desde la perspectiva de la ingeniería documental, debemos señalar el carácter volátil de los documentos $\mathrm{xml}$. Principalmente, éstos constituyen la interfaz entre los procesos y las aplicaciones informáticas que los gestionan; a partir de un mismo repositorio de datos relacionales (diseñado según las técnicas de modelado de datos) pueden generarse distintos documentos $\mathrm{xml}$, que se intercambiarán durante las distintas transacciones y procesos. El documento xml constituye el "sobre" a través del cual se envían unos datos para que resulten comprensibles y sean fácilmente procesables. 
Las técnicas de modelado de datos relacional quedan fuera del alcance de la ingeniería documental, si bien en un proyecto real exigirá aplicar mecanismos que permitan extraer y generar documentos xml a partir de una base de datos relacional, ser capaces de comprender estas técnicas de modelado y establecer correspondencias entre los elementos que forman los documentos $\mathrm{xml}$ y las estructuras utilizadas en el modelo de datos origen.

\section{Documentos orientados a transacciones}

Los tipos de documentos que se pretenden diseñar son los destinados a ser usados por transacciones automatizadas. La ingeniería documental no considera entre sus objetivos el tratamiento de documentos destinados a la impresión, visualización en pantalla, o a "usuarios humanos".

Los aspectos relativos a la presentación de los documentos xml queda fuera del objetivo de esta disciplina. Las ventajas que ofrece este lenguaje para reutilizar el mismo contenido en múltiples publicaciones, automatizar procesamientos costosos como la generación de índices o volver a publicar un mismo documento en múltiples formatos no son aplicables en los entornos de negocio en los que se centra la ingeniería documental ${ }^{5}$.

La principal diferencia está en el hecho de que, cuando se usa xml como formato de codificación de publicaciones y documentos destinados a personas (el documento $\mathrm{xml}$ es persistente: es decir, se ha creado y codificado con el propósito de permanecer y durar) en estos escenarios, el repositorio de información será una colección de docu- mentos xml gestionados mediante algún sistema de gestión documental.

Frente a esto, el uso de documentos xml en transacciones de comercio electrónico se caracteriza por su naturaleza volátil: se genera con el único fin de facilitar la transmisión de unos datos a través de internet, de forma que estos datos sean fácilmente procesables por la aplicación informática que los recibe. Una vez el documento ha sido recibido y procesado, pierde su razón de existir y deja de ser necesario (tendríamos que matizar este punto, ya que no debemos olvidar la necesidad de auditar la recepción de mensajes). En este escenario, el repositorio de información y datos suele ser una base de datos relacional, y los documentos xml son únicamente un medio para transferir la información.

En palabras de Glushko, el hecho de que los documentos $\mathrm{xml}$ sirvan de interfaz para hacer llegar datos a un sistema detrás del cual se encuentra un modelo de datos relacional, obliga a aplicar en el diseño de documentos xml, "el mismo rigor que caracteriza a las técnicas de modelado de datos relacionales".

\section{Notas}

1. En una entrevista a Ralph Drayer (CIO, 0108-02) (vicepresidente de la División de servicios al cliente de Procter \& Gamble en 1987) describe cómo el sistema vendido a Wal-Mart se basó en un prototipo previo diseñado por $P \& G$ para los supermercados Schnucks, y cómo $P \& G$ también lo ofreció a Kmart (incluso antes que a Wal-Mart), pero éstos no la aceptaron, lo que parece ser tuvo consecuencias en una pérdida de competitividad respecto a Wal-Mart.

2. En la entrevista, Ralph Drayer señala cómo $P \& G$ desarrolló el sistema de suministro a partir de una aplicación de IBM llamada Inform. Posteriormente $P \& G$ lo vendió nuevamente a $I B M$, que comercializó el producto y fijó un estándar para la industria conocido como $C R P$.
3. Los lenguajes dedicados a describir procesos en forma de orquestaciones de servicios web quedan fuera del alcance de este trabajo.

4. Es posible establecer una equivalencia entre las distintas iniciativas que han tratado de identificar un conjunto de componentes comunes, reutilizables en distintos tipos de documentos, y el trabajo de L. Silverston publicado el año 2001: The data model resource book. Esta obra en 2 volúmenes recoge distintos modelos de datos relacionales aplicables a diferentes sectores (para la gestión de datos financieros, información de clientes, compras, ventas, recursos humanos, etc.). $\mathrm{Su}$ aspecto más interesante es que ofrece patrones de diseño comunes y válidos para cualquier organización que tenga que realizar un modelado de datos.

5. Esto no debe malinterpretarse y pensar que, en un momento cualquiera, y en respuesta a las necesidades del proceso de negocio que se quiera implementar, no sea posible automatizar la generación de informes u otras representaciones a partir de la información tramitada mediante estos documentos xml. El hecho de usar formatos estándares como xml haría posible el mismo tipo de tratamiento que se hace con los documentos en los entornos de publicación.

\section{Referencias}

Walsh, Aaron E. (ed.). EbXML: the technical specifications. Upper Saddle River, NJ: Prentice Hall PTR, 2002.

Gibb, Brian; Damodaran, Suresh. EbXML: concepts and application. Indianapolis, IN: Wiley Pub., 2003.

Glushko, Robert. Document Engineering for e-business. University of California, Berkeley, Spring 2002.

http://www.sims.berkeley.edu/academics/courses/is290-4/s02

Glushko, Robert. How xml enables internet trading communities and marketplaces. 2000. http://www.infoloom.com/gcaconfs/WEB

Glushko, Robert. Patterns and reuse in document engineering.

http://www.sims.berkeley.edu/ glushko

Glushko, Robert J.; McGrath, Tim. Document engineering: analyzing and designing documents for business informatics and web services. MIT Press, 2005. 400 p. ISBN 0-262-07261-0

Malone, Thomas W. The future of work. Boston: Harvard Business School Press, 2004.

Nayak, N.; Bhaskaran, K.; Das, R. "Virtual enterprises-building blocks for dynamic e-business". En: Information technology for virtual enterprises, 2001, pp. 80-87.

Ricardo Eito-Brun, Universidad Carlos III de Madrid.

reito@bib.uc3m.es

Leer EPI es como asistir a un curso de formación continua pero con el horario acomodado a tus necesidades. 